\title{
Applications of Mesenchymal Stem Cells and Neural Crest Cells in Craniofacial Skeletal Research
}

\author{
Satoru Morikawa, ${ }^{1,2}$ Takehito Ouchi, ${ }^{1,2}$ Shinsuke Shibata, ${ }^{2}$ Takumi Fujimura, \\ Hiromasa Kawana, ${ }^{1}$ Hideyuki Okano, ${ }^{2}$ and Taneaki Nakagawa ${ }^{1}$ \\ ${ }^{1}$ Department of Dentistry and Oral Surgery, Keio University School of Medicine, 35 Shinanomachi, Shinjuku-ku, \\ Tokyo 160-8582, Japan \\ ${ }^{2}$ Department of Physiology, Keio University School of Medicine, 35 Shinanomachi, Shinjuku-ku, Tokyo 160-8582, Japan \\ Correspondence should be addressed to Satoru Morikawa; morikawa@keio.jp
}

Received 11 December 2015; Accepted 2 February 2016

Academic Editor: Jiabing Fan

Copyright (C) 2016 Satoru Morikawa et al. This is an open access article distributed under the Creative Commons Attribution License, which permits unrestricted use, distribution, and reproduction in any medium, provided the original work is properly cited.

\begin{abstract}
Craniofacial skeletal tissues are composed of tooth and bone, together with nerves and blood vessels. This composite material is mainly derived from neural crest cells (NCCs). The neural crest is transient embryonic tissue present during neural tube formation whose cells have high potential for migration and differentiation. Thus, NCCs are promising candidates for craniofacial tissue regeneration; however, the clinical application of NCCs is hindered by their limited accessibility. In contrast, mesenchymal stem cells (MSCs) are easily accessible in adults, have similar potential for self-renewal, and can differentiate into skeletal tissues, including bones and cartilage. Therefore, MSCs may represent good sources of stem cells for clinical use. MSCs are classically identified under adherent culture conditions, leading to contamination with other cell lineages. Previous studies have identified mouse- and human-specific MSC subsets using cell surface markers. Additionally, some studies have shown that a subset of MSCs is closely related to neural crest derivatives and endothelial cells. These MSCs may be promising candidates for regeneration of craniofacial tissues from the perspective of developmental fate. Here, we review the fundamental biology of MSCs in craniofacial research.
\end{abstract}

\section{Introduction}

Developmental origins are beginning to be elucidated through rigorous studies in stem cell biology. Recent studies have demonstrated that the basis of regenerative medicine can be found in developmental biology. Indeed, many applications in regenerative medicine mimic the development and healing of specific tissues.

Mesenchymal stem cells (MSCs) are commonly used in both basic and clinical studies because they can be easily identified in adult tissues. MSCs were first identified as fibroblast-like cells in the bone marrow [1], resemble colony forming unit-fibroblasts (CFU-Fs) at clonal density, and have the capacity for differentiation into mesenchymal lineages, such as bone, cartilage, and fat [2]. Notably, MSCs and neural crest cells (NCCs) are both used in various approaches in craniofacial biology because of their developmental similarities. Indeed, the craniofacial mesenchyme developmentally originates from NCCs [3-5], and NCCs are developmentally identified at the embryonic stages [6]. It is difficult to isolate NCCs because of their limited accessibility and ethical concerns; therefore, it is difficult to directly use NCCs in patients. In contrast, MSCs are present in easily accessible adult tissues, such as bone marrow, fat tissues, and synovium, enabling facile isolation. Importantly, MSCs and NCCs have similar self-renewal and differentiation potential, and MSCs are capable of differentiating into neuronal cells [7, 8]. Furthermore, MSCs can also differentiate into endothelial cells [9-11] and are indispensable for tissue formation [12, 13]. Similar findings have also been reported in skeletal tissues $[14,15]$, suggesting that adult MSCs may be useful in clinical applications associated with the regeneration of skeletal tissues, particularly because of the necessity for synchronized neural tissue formation and vascularization.

Skeletal stem cells (SSCs), which were recently identified $[16,17]$, have been shown to contribute to the construction 
of skeletal tissues during development and wound healing. However, the formation and regeneration of skeletal tissues involve not only construction of bone, but also vascularization and neural synchronization [18-20]. Despite this fact, few studies have evaluated these processes with regard to SSCs.

NCCs, MSCs, and SSCs are all isolated using culture and exhibit overlapping self-renewal and multipotent differentiation potential. Thus, clarifying the specific characteristics of each cell type will improve the clinical application of these types of stem cells. In this review, we discuss the fundamental biology of stem cells in craniofacial research.

\section{Stem Cells in Craniofacial Research}

Skeletal tissues are composed of a network of hard tissues, including bone and cartilage. The jawbone and teeth comprise the craniofacial region, and many individuals suffer from skeletal diseases, such as metastasis of oral malignant tumors into the bone, congenital craniofacial malformation, severe periodontitis, and medication-related osteonecrosis of the jaw [21]. These diseases cause eating difficulty, aesthetic disorders, respiratory distress, and speech disorders, leading to decreased quality of life. Current fundamental approaches to these diseases include surgery and subsequent reconstruction using artificial materials or xenobiomaterials. However, natural bone formation and healing using autologous cells are preferable. Therefore, development-based medicine and approaches are desired.

During the most recent decade, stem cell research has made great advances in clarifying the mechanisms of tissue development. Indeed, many stem cell researchers have focused on developmental biology and regenerative medicine, and applications of stem cells in craniofacial research have been proposed. In particular, MSCs and NCCs have been studied extensively in craniofacial research. MSCs partially originate from NCCs [7, 22-25]; therefore, some MSCs may also differentiate into neuronal cells [26-28], suggesting that specific subsets of MSCs may have the same potential as NCCs. Moreover, because MSCs are present in several adult tissues [2], they are easy to isolate and expand in vitro.

Notably, dental-specific MSCs have been identified in craniofacial tissues. Several research groups have reported the presence of dental MSCs in dental pulp stem cells (DPSCs) [29], stem cells from exfoliated deciduous teeth (SHED) [30], periodontal ligament stem cells (PDLSCs) [31], and stem cells from apical papilla (SCAP) [32]. These dental MSCs may have applications in degenerative and intractable diseases [33]. Furthermore, conditioned medium (CM) from dental MSCs supplies paracrine factors and may be effective for injured areas [34, 35]. Osugi et al. reported that SHEDCM promoted the growth of bone mass in a calvarial defect model. Additionally, the use of conditioned medium from dental tissue-derived MSCs is a unique approach for craniofacial regenerative medicine without cell transplantation. This approach may reduce costs and time/labor requirements and may alleviate safety concerns [36].
Bone marrow MSCs are utilized as a typical model for clinical studies and basic biology research, and they are classically defined by conventional culture, as MSCs show vigorous expansion and multipotent differentiation. However, the mechanisms of regeneration in conventional MSCs cannot be traced back to developmental fate, and it is difficult to predict which subsets of a crowded cell population contribute to the development of specific target tissues. Thus, mixed cultures of conventional MSCs may not provide consistent, predictable therapeutic outcomes from a developmental biological viewpoint. Further studies are needed to clarify the development and degeneration of craniofacial skeletal tissues using stem cells, particularly for analysis of the clonal phenotype of NCCs and MSCs.

\section{Purified MSCs Are Partially Derived from NCCs}

Cranial NCCs constitute a major part of facial tissues $[3,37]$. Moreover, NCCs are localized in the neural folds of fetal tissues, migrate into various tissues, and regulate skeletal tissues [38]. Cranial NCCs form the first pharyngeal arch innerved by the trigeminal nerve and the second pharyngeal arch innerved by the facial nerve [39-41]. Then, the first and second pharyngeal arches interact with each other and form craniofacial tissues $[42,43]$. Craniofacial tissues are mainly composed of bone, muscle, and tendon together with the neurovascular bundle [44]. Therefore, improving the understanding of neurogenesis and vascularization in skeletal tissues is essential for discussions of craniofacial tissue formation. To identify and track the process of cranial tissue specification, visualization of the neural crest and its derivatives is needed.

Within the last few decades, transgenic technologies have been developed that enable visualization of target cells for prospective and retrospective analyses [45]. Transgenic animal studies can be used to elucidate what and how specific cells contribute to form target tissues. This approach has also been applied to the craniofacial field, where NCCs and their derivative cells are labeled and their fates are analyzed [4, 46, 47]. This transgenic technology provides clear information on the development of NCCs in the craniofacial field and enables the visualization of NCCs and their derivatives within dental and craniofacial tissues as shown in Figure 1(a).

Some researchers have focused on cells that are easier to obtain than embryonic NCCs, such as bone marrow stem cells or pluripotent stem cells. The bone marrow is a typical source of MSCs and contains two distinct types of stem cells, hematopoietic stem cells (HSCs), and MSCs [48]. Transgenic animal studies have demonstrated that MSCs partially originate from NCCs. NCCs delaminate from neuroepithelial cells, which can differentiate into cells expressing the MSC marker platelet-derived growth factor receptor alpha (PDGFR $\alpha)$ (CD140a) [22]. Thus, neuroepithelial cells are a source of MSC differentiation. Moreover, Nagoshi et al. showed that NCCs migrate though the aortagonad-mesonephros region and circulate into the bone marrow, demonstrating how neural crest-derived stem cells can travel to the bone marrow [23]. Additionally, Morikawa 

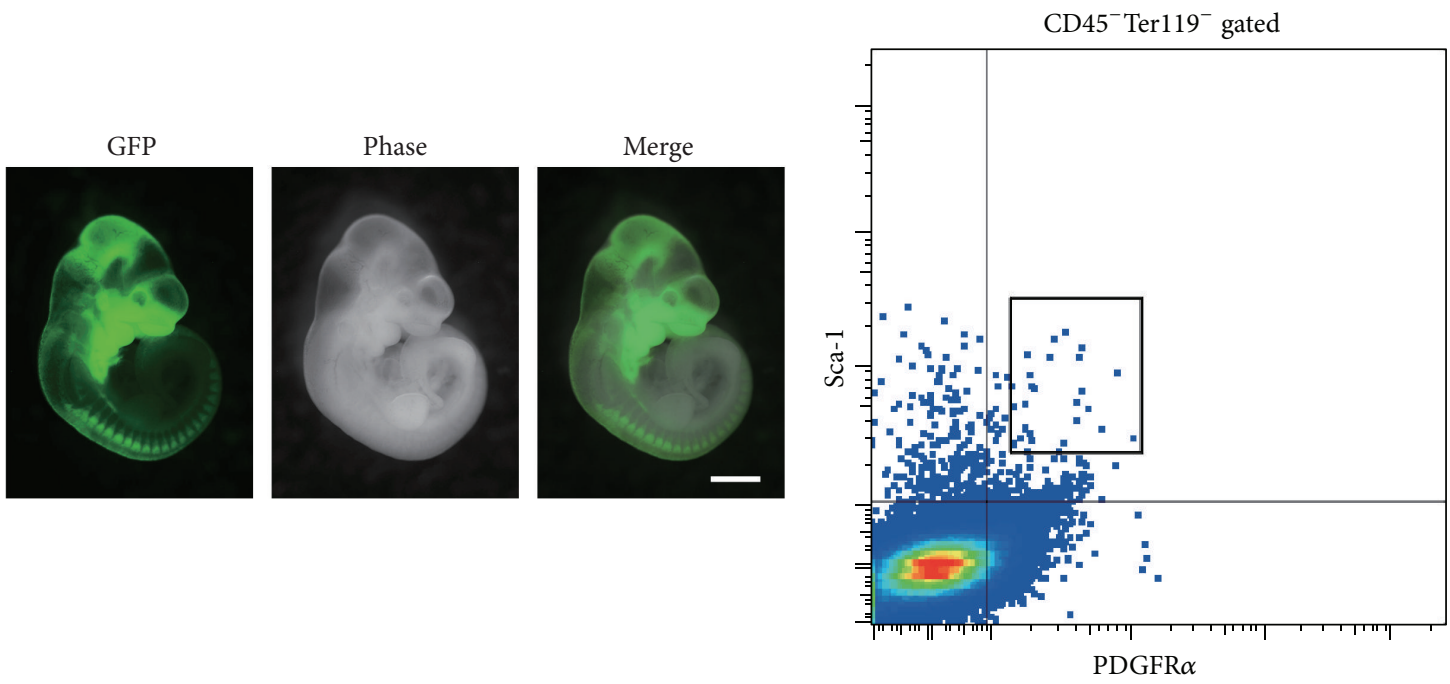

(a)

(b)

Figure 1: (a) Neural crest lineage labeling mouse (Wntl-Cre/GFP) clearly demonstrates green fluorescence-positive NCCs in craniofacial tissues at embryonic day 11. Scale bar, $1 \mathrm{~mm}$. (b) Murine bone marrow cells were analyzed by flow cytometry. The chart shows that cells expressing hematopoietic lineage markers (CD45 and Ter119) were negatively gated, and the highly potent murine MSC marker-expressing $(\mathrm{P} \alpha \mathrm{S})$ subset is indicated by the black-colored box.

et al. reported a subset of highly potent murine MSCs, characterized by the cell surface marker combination of $\operatorname{PDGFR} \alpha$, stem cell antigen-1 (Sca-1/lymphocyte activation protein; Ly-6), CD45, and Ter119 (Ly-76). This specific subset, PDGFR $\alpha^{+}$Sca- $1^{+} \mathrm{CD} 45^{-}$Ter119 ${ }^{-}(\mathrm{P} \alpha \mathrm{S})$, could be found in the bone marrow as shown in Figure 1(b) [49]. The $\mathrm{P} \alpha \mathrm{S}$ subset partially originates from NCCs as shown by developmental fate analysis using transgenic mice [7]. Thus, application of cell tracking systems in mice has clarified the relationship between MSCs and NCCs, supporting the application of MSCs in craniofacial skeletal tissue research.

The $\mathrm{P} \alpha \mathrm{S}$ subset of MSCs has been identified as the perivascular niche and has differentiation potential to both mesenchymal and neural crest lineages [7]. Indeed, the $\mathrm{P} \alpha \mathrm{S}$ subset promotes neural crest-derived periodontal tissue regeneration [50]. $\mathrm{P} \alpha \mathrm{S}$ cells are closely related to SSCs, which are identified by rigorous assays. Bone marrow MSCs also contain stem cells that can only differentiate into skeletal tissues. Thus, SSCs represent a reservoir for bone-forming cells and have the potential to shape and regulate the local microvascular network in the bone marrow [51].

Worthley et al. reported that Gremlin-1 functions as a specific marker of skeletal stem cells in the long bone marrow. Gremlin-1 is a bone morphogenic peptide (BMP) antagonist, and the transforming growth factor (TGF)- $\beta / \mathrm{BMP}$ signaling pathway regulates osteoblastogenesis and bone formation [52]. In Gremlin-1-overexpressing transgenic mice, Gremlin$1^{+}$cells differentiate into bone, cartilage, and reticular stromal cells. Postnatally, Gremlin- $1^{+}$cells also contribute to development. Gremlin $-1^{+}$cells are not further enriched for $\mathrm{P} \alpha \mathrm{S}$ cells. However, the $\mathrm{P} \alpha \mathrm{S}$ subset also contributes to the formation of skeletal tissues [53]. Therefore, some MSCs in the $\mathrm{P} \alpha \mathrm{S}$ subset may have the differentiation potential of SSCs. Consistent with this notion, Gremlin regulates developing limbs, and Gremlin $-1^{+}$and $\mathrm{P} \alpha \mathrm{S}$ cell subsets have been identified in the long bone [17, 49, 54,55]. Additionally, recent reports have shown that cranial MSCs are different from long bone marrow MSCs [56].

Zhao et al. reported that glioma-associated oncogene homolog-1 (Gli-1) is a marker of craniofacial-specific MSCs in cranial bones [57]. Gli-1 ${ }^{+}$cells are not coexpressed with classical MSC markers in vivo. Although the specific characteristics of these cells have not been defined in vivo, Gli- $1^{+}$cells show typical phenotypes of MSCs, such as vigorous expansion, expression of classical MSC markers, and differentiation to mesenchymal lineages in vitro $[44,57]$. However, Gli-1 is not always expressed in MSCs during development, in contrast to PDGFR $\alpha$. Notably, no direct gene regulation mechanism has been identified between Gli-1 and PDGFR $\alpha$ [58]. Gli-1 is induced by sonic hedgehog ( $\mathrm{Shh}$ ) signaling and is associated with transient Sox2 expression during tooth formation [59]. Shh signaling has also been detected in Hertwig's epithelial root sheath (HERS) and found to lead to tooth root formation [60], suggesting that Shh signaling may promote Gli-1 expression in craniofacial-specific mesenchyme. Although Gli-1 ${ }^{+}$ cells are not present in the perivascular niche [57], MSCs in long bones are regulated by the perivascular niche [61-65]. The leptin receptor (LepR) (CD295) has been reported as an excellent marker for MSCs. LepR ${ }^{+}$cells are identified around sinusoids $[61,66]$. In contrast, MSCs in the cranial bone suture are found around the midline of the suture structure. This specificity of craniofacial MSCs does not correspond 
to MSCs in long bone marrow. These differences in murine craniofacial and long bone MSCs should be examined in greater detail in future studies.

\section{Purified MSCs Can Be Derived from Induced Pluripotent Stem Cells (iPSCs)}

To achieve the transition of stem cell research from the bench to the bedside, more studies investigating human cells are required. Human MSCs can be found in several types of tissues. The classical definition of MSCs is the same in mice and in humans, and conventional MSCs can be contaminated by other cell lineages. To avoid such problems, more studies of cell markers and selection of target cells are needed. Previously, Mabuchi et al. identified the highly specific human MSC markers, low-affinity nerve growth factor receptor (LNGFR), and thymocyte antigen 1 (THY-1) [67]. Cells expressing LNGFR and THY-1 have been identified in the bone marrow, and the combination of LNGFR and THY-1 cell surface markers characterizes a distinct subset of MSCs with a hematopoietic lineage. $\mathrm{LNGFR}^{+} \mathrm{THY}^{+}{ }^{+}$cells have high colony forming potential and differentiate into mesenchymal lineages. These specific cells have also been identified in decidua, fat tissues, synovium, and dental pulp [67-69]. The $\mathrm{LNGFR}^{+} \mathrm{THY}^{+}{ }^{+}$subset shows highly potent self-renewal and differentiation capacity both in vitro and in vivo and is also associated with the expression of other classical MSC markers, such as CD29 (integrin beta 1), CD44 (homing cell adhesion molecule; HCAM), CD73 (ecto5'-nucleotidase), CD105 (endoglin), CD146 (melanoma cell adhesion molecule; MCAM), and CD166 (activated leukocyte cell adhesion molecule; ALCAM) [67, 69]. Efficient procedures for purification will improve the ability to analyze MSCs.

In craniofacial diseases, there is a great need for reconstruction of large areas affected by disease or injury. Thus, it is necessary to obtain large numbers of MSCs for such clinical applications. This must be achieved without damaging original tissues; therefore, specific cells derived from pluripotent stem cells based on developmental research may help to overcome this problem.

Human iPSCs were first generated in 2007 and have been used extensively in biomedical studies [70]. Human iPSCs have the capacity for self-renewal and can be expanded relatively easily. The strong potential of iPSCs can also be applied in craniofacial research. MSCs can be derived both directly from iPSCs and indirectly from neural crest like cells using specific markers and culture conditions [24, 71]. MSCs induced from iPSCs have been used in the regeneration of periodontal tissues [72]. Thus, these findings suggest that iPSC-derived MSCs have the capacity for use in craniofacial tissue regeneration.

\section{Application of Human MSCs in Craniofacial Research}

Craniofacial connective tissues originate from neural crestderived ectomesenchyme, which is a source of many craniofacial bone and cartilage structures. Umeda et al. reported the generation of ectomesenchymal cells through neural crestlike progeny from human iPSCs [73]. Sensory innervation is necessary for maintaining sound bone. In dentistry, sensory innervation of craniofacial tissues involves the trigeminal nerve. Previously, several methods were reported for induction of peripheral nerve formation using NCCs [74, 75]. Application of sensory neuronal cells to craniofacial skeletal tissues requires neural crest-derived craniofacial-specific sensory neuronal induction. Dincer et al. reported that the craniofacial placode can be used to identify the craniofacial trigeminal nerve [76]. However, induction procedures for craniofacial target tissues are a relatively new approach in regenerative medicine and disease-specific iPSC technology. Further studies are needed to provide clear information on neural crest biology and craniofacial specificity. Methods for induction of target cells must take advantage of the generation of a sufficient number of cells, and induction based on the basic knowledge of developmental biology may provide an evidence-based approach for application of stem cells in the clinical field.

For application of human iPSC-derived cells in the regeneration of craniofacial tissues, prevention of teratoma formation represents a major challenge. iPSC lines show variations in the patterns of teratoma formation in iPSC-derived neural progenitor cells $[77,78]$. Surprisingly, despite the exclusion of pluripotent markers, iPSCs may form teratomas in some cases. Lee et al. reported that iPSC-derived neural crest-derived stem cells, which exhibit downregulation of polysialic acid-neural cell adhesion molecule (PSA-NCAM), tend to form teratomas [79]. These findings suggest the importance of basic and preclinical studies of iPSC-derived NCCs. Recent studies have shown the safety of iPSC-derived cells in preclinical models [80]. Reconstruction of the target craniofacial tissues in nonhuman primate models is essential before human clinical studies using iPSCs can be initiated. Prescott et al. showed that cis-regulatory divergence is associated with differences in quantitative expression in human and chimpanzee cranial NCCs derived from iPSCs [81]. It is quite important that this study demonstrated the novel application of iPSC-derived NCCs for analyzing evolutionary cellular anthropology in the context of craniofacial development.

Thus, iPSC technology may facilitate future applications in regenerative medicine if the risk of iPSC-derived tumor formation can be minimized.

\section{Conclusion}

Skeletal tissues are composed of bone, cartilage, and tendon. These mesenchymal tissues are generated from NCC-derived MSCs and exhibit neurogenesis and neovascularization. MSCs are part of the perivascular niche and overlap with neural crest-derived stem cells [25]. MSCs have potential for differentiating into skeletal cells, neuronal cells, and endothelial cells, suggesting that MSCs may be useful for craniofacial tissue regeneration. Indeed, MSCs have been applied for the treatment of craniofacial diseases, such as periodontitis and osteonecrosis of the jaw in small animal models [82, 83]. The potential of stem cells is typically demonstrated using mice and rats because they are easy to breed and handle, and there 
is a variety of well-established disease models recognized by the scientific community. However, the craniofacial anatomy of these animals is different from that of humans. Several research groups reported stem cell approaches using larger animals such as pigs, dogs, and chimpanzees [32, 81, 84]. In animal studies, establishment of live cell imaging systems can be used to clearly visualize the potential for migration and differentiation. Such imaging systems have been utilized in the craniofacial field $[69,85]$. Demonstration of cell tracking in large animal models such as nonhuman primates provides essential evidence for human preclinical studies that expected to take place in the near future.

Human clinical application of stem cells has already started [86, 87]; however, the current protocols for clinical application mainly utilize conventionally cultured cells. Conventional MSCs contain various types of cells within adherent culture, resulting in contamination of the MSCs with other cell lineages. Moreover, MSCs show great differences in characteristics between long bones and craniofacial tissue, and these differences should be evaluated in detail in future studies.

For further analyses of the applications of MSCs in humans, the developmental fate of human MSCs must be elucidated. Sufficient quantities of purified MSCs from adult tissues for reconstruction of large spaces in the craniofacial region are difficult to collect. Human iPSC technology may be used to overcome this problem. Furthermore, more analyses of MSCs, NCCs, SSCs, and iPSCs are required based on a developmental biological approach, which will be expected to provide evidence-based methods for the treatment of various craniofacial diseases.

\section{Conflict of Interests}

Hideyuki Okano is a paid scientific advisor of San Bio Co. Ltd. The other authors have no conflict of interests to declare.

\section{Authors' Contribution}

Satoru Morikawa and Takehito Ouchi contributed equally to this work.

\section{Acknowledgments}

The authors thank the members of their laboratory for helpful discussions. This work was supported by Japan Society for the Promotion of Science KAKENHI grants numbered 15K11221 (Taneaki Nakagawa) and 25463226 (Satoru Morikawa) and by the Program for Intractable Disease Research Utilizing Disease-specific iPS Cells from the Japan Science and Technology Agency (JST), Japan Agency for Medical Research and Development (A-MED), and the Ministry of Education, Science, Sports and Culture (MEXT) to Hideyuki Okano.

\section{References}

[1] A. J. Friedenstein, U. F. Deriglasova, N. N. Kulagina et al., "Precursors for fibroblasts in different populations of hematopoietic cells as detected by the in vitro colony assay method," Experimental Hematology, vol. 2, no. 2, pp. 83-92, 1974.
[2] M. F. Pittenger, A. M. Mackay, S. C. Beck et al., "Multilineage potential of adult human mesenchymal stem cells," Science, vol. 284, no. 5411, pp. 143-147, 1999.

[3] Y. Chai, X. Jiang, Y. Ito et al., "Fate of the mammalian cranial neural crest during tooth and mandibular morphogenesis," Development, vol. 127, no. 8, pp. 1671-1679, 2000.

[4] K. Hagiwara, T. Obayashi, N. Sakayori et al., "Molecular and cellular features of murine craniofacial and trunk neural crest cells as stem cell-like cells," PLoS ONE, vol. 9, no. 1, Article ID e84072, 2014.

[5] S. Wiszniak, F. E. Mackenzie, P. Anderson, S. Kabbara, C. Ruhrberg, and Q. Schwarz, "Neural crest cell-derived VEGF promotes embryonic jaw extension," Proceedings of the National Academy of Sciences, vol. 112, no. 19, pp. 6086-6091, 2015.

[6] S. J. Morrison, P. M. White, C. Zock, and D. J. Anderson, "Prospective identification, isolation by flow cytometry, and in vivo self-renewal of multipotent mammalian neural crest stem cells," Cell, vol. 96, no. 5, pp. 737-749, 1999.

[7] S. Morikawa, Y. Mabuchi, K. Niibe et al., "Development of mesenchymal stem cells partially originate from the neural crest," Biochemical and Biophysical Research Communications, vol. 379, no. 4, pp. 1114-1119, 2009.

[8] A. Arthur, G. Rychkov, S. Shi, S. A. Koblar, and S. Gronthose, "Adult human dental pulp stem cells differentiate toward functionally active neurons under appropriate environmental cues," STEM CELLS, vol. 26, no. 7, pp. 1787-1795, 2008.

[9] K. Janeczek Portalska, A. Leferink, N. Groen et al., "Endothelial differentiation of mesenchymal stromal cells," PLoS ONE, vol. 7, no. 10, Article ID e46842, 2012.

[10] G. V. Silva, S. Litovsky, J. A. R. Assad et al., "Mesenchymal stem cells differentiate into an endothelial phenotype, enhance vascular density, and improve heart function in a canine chronic ischemia model," Circulation, vol. 111, no. 2, pp. 150-156, 2005.

[11] J. Oswald, S. Boxberger, B. Jørgensen et al., "Mesenchymal stem cells can be differentiated into endothelial cells in vitro," STEM CELLS, vol. 22, no. 3, pp. 377-384, 2004.

[12] T. Takebe, M. Enomura, E. Yoshizawa et al., "Vascularized and complex organ buds from diverse tissues via mesenchymal celldriven condensation," Cell Stem Cell, vol. 16, no. 5, pp. 556-565, 2015.

[13] T. Takebe, K. Sekine, M. Enomura et al., "Vascularized and functional human liver from an iPSC-derived organ bud transplant," Nature, vol. 499, no. 7459, pp. 481-484, 2013.

[14] S. Kobayashia, T. Takebe, M. Inui et al., "Reconstruction of human elastic cartilage by a $\mathrm{CD} 44^{+} \mathrm{CD} 90^{+}$stem cell in the ear perichondrium," Proceedings of the National Academy of Sciences of the United States of America, vol. 108, no. 35, pp. 14479-14484, 2011.

[15] T. Takebe, S. Kobayashi, H. Suzuki et al., "Transient vascularization of transplanted human adult-derived progenitors promotes self-organizing cartilage," The Journal of Clinical Investigation, vol. 124, no. 10, pp. 4325-4334, 2014.

[16] C. K. F. Chan, E. Y. Seo, J. Y. Chen et al., "Identification and specification of the mouse skeletal stem cell," Cell, vol. 160, no. 1-2, pp. 285-298, 2015.

[17] D. L. Worthley, M. Churchill, J. T. Compton et al., "Gremlin 1 identifies a skeletal stem cell with bone, cartilage, and reticular stromal potential," Cell, vol. 160, no. 1-2, pp. 269-284, 2015.

[18] T. Fukuda, S. Takeda, R. Xu et al., "Sema3A regulates bonemass accrual through sensory innervations," Nature, vol. 497, no. 7450, pp. 490-493, 2013. 
[19] T. Tamaki, Y. Uchiyama, Y. Okada et al., "Functional recovery of damaged skeletal muscle through synchronized vasculogenesis, myogenesis, and neurogenesis by muscle-derived stem cells," Circulation, vol. 112, no. 18, pp. 2857-2866, 2005.

[20] S. Levenberg, J. Rouwkema, M. Macdonald et al., "Engineering vascularized skeletal muscle tissue," Nature Biotechnology, vol. 23, no. 7, pp. 879-884, 2005.

[21] A. O. M. Wilkie and G. M. Morriss-Kay, "Genetics of craniofacial development and malformation," Nature Reviews Genetics, vol. 2, no. 6, pp. 458-468, 2001.

[22] Y. Takashima, T. Era, K. Nakao et al., "Neuroepithelial cells supply an initial transient wave of MSC differentiation," Cell, vol. 129, no. 7, pp. 1377-1388, 2007.

[23] N. Nagoshi, S. Shibata, Y. Kubota et al., "Ontogeny and multipotency of neural crest-derived stem cells in mouse bone marrow, dorsal root ganglia, and whisker pad," Cell Stem Cell, vol. 2, no. 4, pp. 392-403, 2008.

[24] M. Fukuta, Y. Nakai, K. Kirino et al., "Derivation of mesenchymal stromal cells from pluripotent stem cells through a neural crest lineage using small molecule compounds with defined media," PLoS ONE, vol. 9, no. 12, Article ID e112291, 2014.

[25] J. Isern, A. García-García, A. M. Martín et al., "The neural crest is a source of mesenchymal stem cells with specialized hematopoietic stem cell niche function," eLife, vol. 3, Article ID e03696, 2014.

[26] J. Kohyama, H. Abe, T. Shimazaki et al., "Brain from bone: efficient 'meta-differentiation' of marrow stroma-derived mature osteoblasts to neurons with Noggin or a demethylating agent," Differentiation, vol. 68, no. 4-5, pp. 235-244, 2001.

[27] T. Tondreau, M. Dejeneffe, N. Meuleman et al., "Gene expression pattern of functional neuronal cells derived from human bone marrow mesenchymal stromal cells," BMC Genomics, vol. 9, article 166, 2008.

[28] Y. S. Takeda and Q. Xu, "Neuronal differentiation of human mesenchymal stem cells using exosomes derived from differentiating neuronal cells," PLoS ONE, vol. 10, no. 8, Article ID e0135111, 2015.

[29] S. Gronthos, M. Mankani, J. Brahim, P. G. Robey, and S. Shi, "Postnatal human dental pulp stem cells (DPSCs) in vitro and in vivo," Proceedings of the National Academy of Sciences of the United States of America, vol. 97, no. 25, pp. 13625-13630, 2000.

[30] M. Miura, S. Gronthos, M. Zhao et al., "SHED: stem cells from human exfoliated deciduous teeth," Proceedings of the National Academy of Sciences of the United States of America, vol. 100, no. 10, pp. 5807-5812, 2003.

[31] B. M. Seo, M. Miura, S. Gronthos et al., "Investigation of multipotent postnatal stem cells from human periodontal ligament," The Lancet, vol. 364, no. 9429, pp. 149-155, 2004.

[32] W. Sonoyama, Y. Liu, D. Fang et al., "Mesenchymal stem cellmediated functional tooth regeneration in swine," PLOS ONE, vol. 1, article e79, 2006.

[33] P. De Berdt, J. Vanacker, B. Ucakar et al., "Dental apical papilla as therapy for spinal cord injury," Journal of Dental Research, vol. 94, no. 11, pp. 1575-1581, 2015.

[34] K. Sakai, A. Yamamoto, K. Matsubara et al., "Human dental pulp-derived stem cells promote locomotor recovery after complete transection of the rat spinal cord by multiple neuroregenerative mechanisms," The Journal of Clinical Investigation, vol. 122, no. 1, pp. 80-90, 2012.

[35] S. Yamaguchi, R. Shibata, N. Yamamoto et al., "Dental pulpderived stem cell conditioned medium reduces cardiac injury following ischemia-reperfusion," Scientific Reports, vol. 5, Article ID 16295, 2015.

[36] M. Osugi, W. Katagiri, R. Yoshimi, T. Inukai, H. Hibi, and M. Ueda, "Conditioned media from mesenchymal stem cells enhanced bone regeneration in rat calvarial bone defects," Tissue Engineering Part A, vol. 18, no. 13-14, pp. 1479-1489, 2012.

[37] F. Santagati, M. Minoux, S.-Y. Ren, and F. M. Rijli, “Temporal requirement of Hoxa2 in cranial neural crest skeletal morphogenesis," Development, vol. 132, no. 22, pp. 4927-4936, 2005.

[38] A. T. Van Ho, S. Hayashi, D. Bröhl, F. Auradé, R. Rattenbach, and F. Relaix, "Neural crest cell lineage restricts skeletal muscle progenitor cell differentiation through Neuregulin1-ErbB3 signaling," Developmental Cell, vol. 21, no. 2, pp. 273-287, 2011.

[39] S. Shibata, A. Yasuda, F. Renault-Mihara et al., "Sox10-Venus mice: a new tool for real-time labeling of neural crest lineage cells and oligodendrocytes," Molecular Brain, vol. 3, no. 1, article 31, 2010.

[40] H. Sato, M. Shibata, T. Shimizu et al., "Differential cellular localization of antioxidant enzymes in the trigeminal ganglion," Neuroscience, vol. 248, pp. 345-358, 2013.

[41] C. Niederländer and A. Lumsden, "Late emigrating neural crest cells migrate specifically to the exit points of cranial branchiomotor nerves," Development, vol. 122, no. 8, pp. 23672374, 1996.

[42] M. Gendron-Maguire, M. Mallo, M. Zhang, and T. Gridley, "Hoxa-2 mutant mice exhibit homeotic transformation of skeletal elements derived from cranial neural crest," Cell, vol. 75, no. 7, pp. 1317-1331, 1993.

[43] H. Zhao, P. Bringas Jr., and Y. Chai, "An in vitro model for characterizing the post-migratory cranial neural crest cells of the first branchial arch," Developmental Dynamics, vol. 235, no. 5, pp. 1433-1440, 2006.

[44] H. Zhao, J. Feng, K. Seidel et al., "Secretion of shh by a neurovascular bundle niche supports mesenchymal stem cell homeostasis in the adult mouse incisor," Cell Stem Cell, vol. 14, no. 2, pp. 160-173, 2014.

[45] P. S. Danielian, D. Muccino, D. H. Rowitch, S. K. Michael, and A. P. McMahon, "Modification of gene activity in mouse embryos in utero by a tamoxifen-inducible form of Cre recombinase," Current Biology, vol. 8, no. 24, pp. 1323-1326, 1998.

[46] N. Kaukua, M. K. Shahidi, C. Konstantinidou et al., "Glial origin of mesenchymal stem cells in a tooth model system," Nature, vol. 513, no. 7519, pp. 551-554, 2014.

[47] I.-H. Chung, T. Yamaza, H. Zhao, P.-H. Choung, S. Shi, and Y. Chai, "Stem cell property of postmigratory cranial neural crest cells and their utility in alveolar bone regeneration and tooth development," STEM CELLS, vol. 27, no. 4, pp. 866-877, 2009.

[48] Y. Koide, S. Morikawa, Y. Mabuchi et al., "Two distinct stem cell lineages in murine bone marrow," Stem Cells, vol. 25, no. 5, pp. 1213-1221, 2007.

[49] S. Morikawa, Y. Mabuchi, Y. Kubota et al., "Prospective identification, isolation, and systemic transplantation of multipotent mesenchymal stem cells in murine bone marrow," Journal of Experimental Medicine, vol. 206, no. 11, pp. 2483-2496, 2009.

[50] Y. Kimura, M. Komaki, K. Iwasaki, M. Sata, Y. Izumi, and I. Morita, "Recruitment of bone marrow-derived cells to periodontal tissue defects," Frontiers in Cell and Developmental Biology, vol. 2, article 19, 2014.

[51] P. Bianco and P. G. Robey, "Skeletal stem cells," Development, vol. 142, no. 6, pp. 1023-1027, 2015. 
[52] M. S. Rahman, N. Akhtar, H. M. Jamil, R. S. Banik, and S. M. Asaduzzaman, "TGF- $\beta /$ BMP signaling and other molecular events: regulation of osteoblastogenesis and bone formation," Bone Research, vol. 3, Article ID 15005, 2015.

[53] N. Ono, W. Ono, T. Nagasawa, and H. M. Kronenberg, "A subset of chondrogenic cells provides early mesenchymal progenitors in growing bones," Nature Cell Biology, vol. 16, no. 12, pp. 11571167, 2014.

[54] R. Merino, J. Rodriguez-Leon, D. Macias, Y. Gañan, A. N. Economides, and J. M. Hurle, "The BMP antagonist Gremlin regulates outgrowth, chondrogenesis and programmed cell death in the developing limb," Development, vol. 126, no. 23, pp. 5515-5522, 1999.

[55] A. Greenbaum, Y.-M. S. Hsu, R. B. Day et al., "CXCL12 in early mesenchymal progenitors is required for haematopoietic stemcell maintenance," Nature, vol. 495, no. 7440, pp. 227-230, 2013.

[56] H. Zhao and Y. Chai, "Stem cells in teeth and craniofacial bones," Journal of Dental Research, vol. 94, no. 11, pp. 1495-1501, 2015.

[57] H. Zhao, J. Feng, T. Ho, W. Grimes, M. Urata, and Y. Chai, "The suture provides a niche for mesenchymal stem cells of craniofacial bones," Nature Cell Biology, vol. 17, no. 4, pp. 386396, 2015.

[58] X.-Q. Zhang, G. B. Afink, X.-R. Hu, K. Forsberg-Nilsson, and M. Nistér, "Glil is not required for Pdgfr $\alpha$ expression during mouse embryonic development," Differentiation, vol. 73, no. 23, pp. 109-119, 2005.

[59] J. Li, J. Feng, Y. Liu et al., "BMP-SHH signaling network controls epithelial stem cell fate via regulation of its niche in the developing tooth," Developmental Cell, vol. 33, no. 2, pp. 125-135, 2015.

[60] Y. Liu, J. Feng, J. Li, H. Zhao, T. Ho, and Y. Chai, "An Nfichedgehog signaling cascade regulates tooth root development," Development, vol. 142, no. 19, pp. 3374-3382, 2015.

[61] B. O. Zhou, R. Yue, M. M. Murphy, J. G. Peyer, and S. J. Morrison, "Leptin-receptor-expressing mesenchymal stromal cells represent the main source of bone formed by adult bone marrow," Cell Stem Cell, vol. 15, no. 2, pp. 154-168, 2014.

[62] M. Crisan, S. Yap, L. Casteilla et al., "A perivascular origin for mesenchymal stem cells in multiple human organs," Cell Stem Cell, vol. 3, no. 3, pp. 301-313, 2008.

[63] B. Sacchetti, A. Funari, S. Michienzi et al., "Self-renewing osteoprogenitors in bone marrow sinusoids can organize a hematopoietic microenvironment," Cell, vol. 131, no. 2, pp. 324336, 2007.

[64] J. Street, M. Bao, L. DeGuzman et al., "Vascular endothelial growth factor stimulates bone repair by promoting angiogenesis and bone turnover," Proceedings of the National Academy of Sciences of the United States of America, vol. 99, no. 15, pp. 9656$9661,2002$.

[65] N. Ono, W. Ono, T. Mizoguchi, T. Nagasawa, P. S. Frenette, and H. M. Kronenberg, "Vasculature-associated cells expressing nestin in developing bones encompass early cells in the osteoblast and endothelial lineage," Developmental Cell, vol. 29, no. 3, pp. 330-339, 2014.

[66] Y. Matsuzaki, Y. Mabuchi, and H. Okano, "Leptin receptor makes its mark on MSCs," Cell Stem Cell, vol. 15, no. 2, pp. 112114, 2014.

[67] Y. Mabuchi, S. Morikawa, S. Harada et al., "LNGFR ${ }^{+}$THY$1^{+}$VCAM- ${ }^{\text {hi+ }}$ cells reveal functionally distinct subpopulations in mesenchymal stem cells," Stem Cell Reports, vol. 1, no. 2, pp. 152-165, 2013.
[68] Y. Ogata, Y. Mabuchi, M. Yoshida et al., "Purified human synovium mesenchymal stem cells as a good resource for cartilage regeneration," PLoS ONE, vol. 10, no. 6, Article ID e0129096, 2015.

[69] T. Yasui, Y. Mabuchi, H. Toriumi et al., "Purified human dental pulp stem cells promote osteogenic regeneration," Journal of Dental Research, vol. 95, no. 2, pp. 206-214, 2016.

[70] K. Takahashi, K. Tanabe, M. Ohnuki et al., "Induction of pluripotent stem cells from adult human fibroblasts by defined factors," Cell, vol. 131, no. 5, pp. 861-872, 2007.

[71] M. Giuliani, N. Oudrhiri, Z. M. Noman et al., "Human mesenchymal stem cells derived from induced pluripotent stem cells down-regulate NK-cell cytolytic machinery," Blood, vol. 118, no. 12, pp. 3254-3262, 2011.

[72] K. Hynes, D. Menicanin, J. Han et al., "Mesenchymal stem cells from iPS cells facilitate periodontal regeneration," Journal of Dental Research, vol. 92, no. 9, pp. 833-839, 2013.

[73] K. Umeda, H. Oda, Q. Yan et al., "Long-term expandable SOX9+ chondrogenic ectomesenchymal cells from human pluripotent stem cells," Stem Cell Reports, vol. 4, no. 4, pp. 712-726, 2015.

[74] G. Lee, H. Kim, Y. Elkabetz et al., "Isolation and directed differentiation of neural crest stem cells derived from human embryonic stem cells," Nature Biotechnology, vol. 25, no. 12, pp. 1468-1475, 2007.

[75] L. Menendez, T. A. Yatskievych, P. B. Antin, and S. Dalton, "Wnt signaling and a Smad pathway blockade direct the differentiation of human pluripotent stem cells to multipotent neural crest cells," Proceedings of the National Academy of Sciences of the United States of America, vol. 108, no. 48, pp. 19240-19245, 2011.

[76] Z. Dincer, J. Piao, L. Niu et al., "Specification of functional cranial placode derivatives from human pluripotent stem cells," Cell Reports, vol. 5, no. 5, pp. 1387-1402, 2013.

[77] K. Miura, Y. Okada, T. Aoi et al., "Variation in the safety of induced pluripotent stem cell lines," Nature Biotechnology, vol. 27, no. 8, pp. 743-745, 2009.

[78] H. Okano, M. Nakamura, K. Yoshida et al., "Steps toward safe cell therapy using induced pluripotent stem cells," Circulation Research, vol. 112, no. 3, pp. 523-533, 2013.

[79] D. R. Lee, J. Yoo, J. Lee et al., "PSA-NCAM-negative neural crest cells emerging during neural induction of pluripotent stem cells cause mesodermal tumors and unwanted grafts," Stem Cell Reports, vol. 4, no. 5, pp. 821-834, 2015.

[80] Y. Kobayashi, Y. Okada, G. Itakura et al., "Pre-evaluated safe human iPSC-derived neural stem cells promote functional recovery after spinal cord injury in common marmoset without tumorigenicity," PLoS ONE, vol. 7, no. 12, Article ID e52787, 2012.

[81] S. L. Prescott, R. Srinivasan, M. C. Marchetto et al., "Enhancer divergence and cis-regulatory evolution in the human and chimp neural crest," Cell, vol. 163, no. 1, pp. 68-83, 2015.

[82] H. Yang, R. M. Aprecio, X. Zhou et al., "Therapeutic effect of TSG-6 engineered iPSC-derived MSCs on experimental periodontitis in rats: a pilot study," PLoS ONE, vol. 9, no. 6, Article ID e100285, 2014.

[83] K. Ogata, W. Katagiri, M. Osugi et al., "Evaluation of the therapeutic effects of conditioned media from mesenchymal stem cells in a rat bisphosphonate-related osteonecrosis of the jaw-like model," Bone, vol. 74, pp. 95-105, 2015.

[84] T. Iwata, M. Yamato, H. Tsuchioka et al., "Periodontal regeneration with multi-layered periodontal ligament-derived cell sheets in a canine model," Biomaterials, vol. 30, no. 14, pp. 2716$2723,2009$. 
[85] J. Dixon, N. C. Jones, L. L. Sandell et al., "Tcof1/Treacle is required for neural crest cell formation and proliferation deficiencies that cause craniofacial abnormalities," Proceedings of the National Academy of Sciences of the United States of America, vol. 103, no. 36, pp. 13403-13408, 2006.

[86] P. Voss, S. Sauerbier, M. Wiedmann-Al-Ahmad et al., "Bone regeneration in sinus lifts: comparing tissue-engineered bone and iliac bone," British Journal of Oral and Maxillofacial Surgery, vol. 48, no. 2, pp. 121-126, 2010.

[87] M. Griffin, D. M. Kalaskar, P. E. Butler, and A. M. Seifalian, "The use of adipose stem cells in cranial facial surgery," Stem Cell Reviews and Reports, vol. 10, no. 5, pp. 671-685, 2014. 

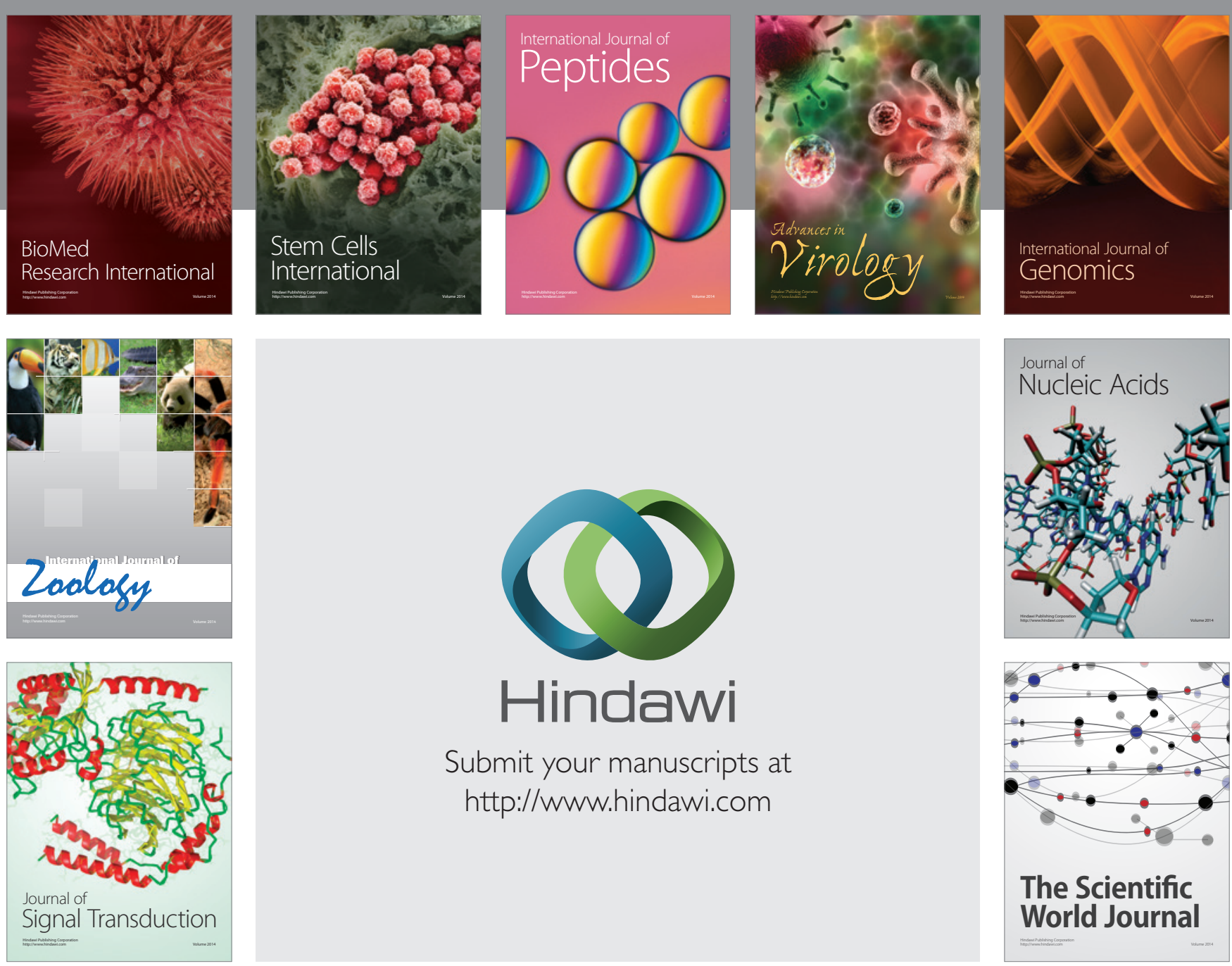

Submit your manuscripts at

http://www.hindawi.com
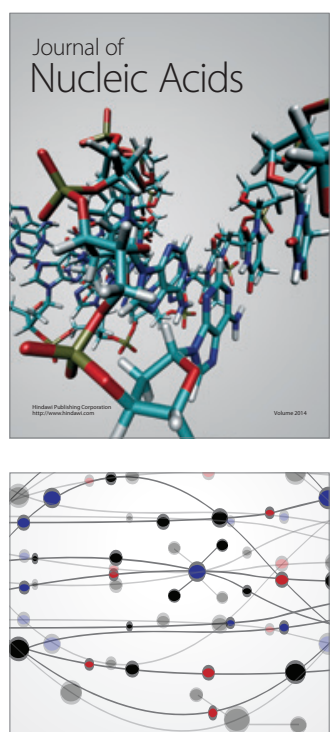

The Scientific World Journal
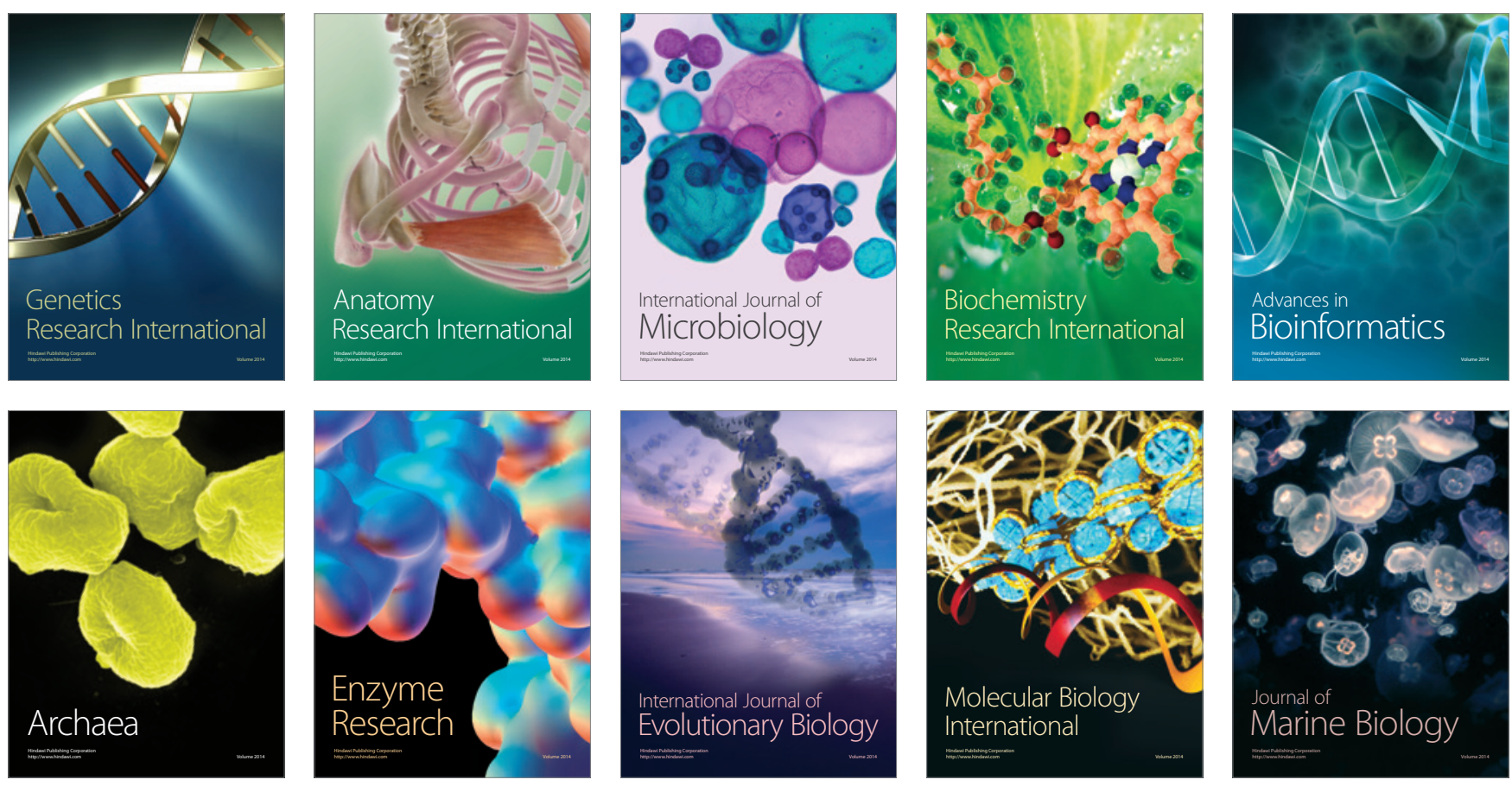